\title{
Distributed chance-constrained optimal power flow based on primary frequency control
}

\author{
Maxime Velay ${ }^{*}$ \\ CEA, LIST, Laboratoire d'Analyse des Donnèes et \\ d'Intelligence des Systèmes \\ Gif-sur-Yvette, France \\ maxime.velay@cea.fr/@g2elab.grenoble-inp.fr \\ Yvon Besanger \\ Univ. Grenoble Alpes, CNRS, Grenoble INP ${ }^{0}$, G2Elab \\ Grenoble, France \\ yvon.besanger@g2elab.grenoble-inp.fr
}

\author{
Meritxell Vinyals \\ CEA, LIST, Laboratoire d'Analyse des Donnèes et \\ d'Intelligence des Systèmes \\ Gif-sur-Yvette, France \\ meritxell.vinyals@cea.fr \\ Nicolas Retiere \\ Univ. Grenoble Alpes, CNRS, Grenoble INP ${ }^{0}$, G2Elab \\ Grenoble, France \\ nicolas.retiere@univ-grenoble-alpes.fr
}

\begin{abstract}
We propose a fully distributed algorithm to solve the Chance Constrained Optimal Power Flow (CCOPF), with the advantages of ensuring the privacy and autonomy of the different operators and actors of the system. We present, in this paper, a two-step algorithm that, first, carries out a distributed sensitivity analysis to obtain the generalized generation distribution factors. With these sensitivity factors, the second step solves a distributed CCOPF based on an analytical formulation relying on the Primary Frequency Control (PFC) of generators and on wind farms, whose forecast errors are assumed to be Gaussian. This algorithm allows us to schedule margins and reserves to ensure the security of the system regarding wind farms deviation from forecast with probabilistic guarantees, and to assess the cost of this uncertainty. The proposed method has been implemented and tested on a two-bus test system with one wind farm, and on the IEEE 14-bus test system, with two wind farms. Simulation results showed that the proposed algorithm can efficiently solve the CCOPF in a fully distributed manner.
\end{abstract}

\section{CCS CONCEPTS}

\section{- Computing methodologies $\rightarrow$ Multi-agent systems;}

\section{KEYWORDS}

Chance-constrained, OPF, ADMM, uncertainty, reserves

\section{ACM Reference Format:}

Maxime Velay, Meritxell Vinyals, Yvon Besanger, and Nicolas Retiere. 2018. Distributed chance-constrained optimal power flow based on primary frequency control. In e-Energy '18: The Nineth International Conference on Future Energy Systems, June 12-15, 2018, Karlsruhe, Germany. ACM, New York, NY, USA, 9 pages. https://doi.org/10.1145/3208903.3208921

\footnotetext{
*Also with Univ. Grenoble Alpes, CNRS, Grenoble INP ${ }^{0}$, G2Elab.

Permission to make digital or hard copies of all or part of this work for personal or classroom use is granted without fee provided that copies are not made or distributed for profit or commercial advantage and that copies bear this notice and the full citation on the first page. Copyrights for components of this work owned by others than the author(s) must be honored. Abstracting with credit is permitted. To copy otherwise, or republish, to post on servers or to redistribute to lists, requires prior specific permission and/or a fee. Request permissions from permissions@acm.org.

e-Energy '18, June 12-15, 2018, Karlsruhe, Germany

(C) 2018 Copyright held by the owner/author(s). Publication rights licensed to ACM ACM ISBN 978-1-4503-5767-8/18/06 . \$ \$15.00

https://doi.org/10.1145/3208903.3208921
}

\section{INTRODUCTION}

Traditionally, power grids were designed to handle energy flows from predictable, controllable and centralized power generation units to final consumers. Hence, the growing integration of renewable energy presents a significant challenge to classical grid operation due to the unpredictable, uncontrollable and highly variable nature of the wind. In fact, under large-scale wind generation, the Optimum Power Flow (OPF), which is solved in a daily basis by the transmission system operators (TSOs) to re-dispatch controllable generation at minimum cost while respecting network constraints (e.g. maximum line capacities and generators), can result in grid instability, and, potentially, in cascading outages. This risk arises because OPF dispatch is computed without taking into account the uncertainty on wind power production (i.e. without considering potential deviations between the forecast and the actual production value). In practice, any deviation leading to power imbalance will be compensated in real-time by the automatic primary frequency response of controllable generators. Since primary generator responses to wind power deviation from forecast are not considered when solving the OPF, this increment of generation can lead to power flows that significantly exceed transmission line limits and, potentially, to their tripping.

Recent works have proposed the chance-constrained optimal power flow (CCOPF) to account for this uncertainty and obtain a schedule that respects network constraints with a high probability, as in [2]. Broadly speaking, chance-constrained optimization is a methodology in which the system security constraints can be violated with a small predefined level of probability. In general, chance-constrained optimization problems are computationally intractable since they require the computation of multi-dimensional probability integrals.

To overcome this difficulty, a solution consists in assuming that the forecast error of each wind farm follows a (previously known) Gaussian probability distribution and that they are mutually independent, as in $[2,11,17]$. The Gaussianity assumption allows for an analytical reformulation of the chance constraints, leading to a deterministic problem with lower computational complexity than scenario based approaches [14]. The mentioned references

\footnotetext{
${ }^{0}$ Institute of Engineering Univ. Grenoble Alpes
} 
$[2,11,17]$ propose centralized approaches to solve the CCOPF problems which are very well suited for single regional area systems. Roald et al. proposed in [17] an exact reformulation of the security constrained OPF with chance constraints related to the uncertainty on the wind power forecast, and under the assumption of independent Gaussian distributions. Similarly, Bienstock et al. [2] formulate the CCOPF and propose an efficient cutting-plane algorithm that proved to be able to solve large problems.

To achieve better overall reliability and economical efficiency, power systems are interconnected and these advantages are subject to have an effective cooperation and coordination among TSOs managing different regions. Unfortunately, the application of centralized approaches in such large-scale interconnected networks is undesirable if not impossible due to [20]: the computational and communication burden of gathering data and taking decisions for the whole system at a central controller; and the privacy issues of having TSOs and generation companies disclosing potentially sensitive information and/or strategic models. Against these drawbacks, distributed methods are gaining popularity since they can preserve the independence of regional operators while fully taking advantage of their interconnection (see $[8,20]$ for a complete review on distributed approaches). In this context, the Alternating Direction Method of Multipliers (ADMM) has attracted increasing attention due to its natural decomposition, its full distributivity and its improved convergence compared to other state-of-the-art distributed optimization algorithms. In recent years, ADMM-based methods have been applied to a wide variety of large-scale power system optimization problems. More precisely, our work is on the track of extension of the works on OPF problems from $[10,18]$ and of SCOPF problem from [19].

Nevertheless, all these above references do not account for any source of uncertainty. Some works $[12,13]$ accounted for wind uncertainty when solving an OPF like problem with ADMM but based on a robust optimization approach. Unlike probabilistic approaches, robust optimization does not require prior knowledge of the underlying probability distribution but instead capture randomness by means of uncertainty sets, which can be overly conservative. Closer to our approach, Hassan et al. also proposes, in [7], a chanceconstrained ADMM approach to minimize power losses and where actuators are the active and reactive power injections of inverterbased distributed energy resources.

To the best of our knowledge, this paper proposes the first CCOPF distributed solution based on the ADMM that relies on an exact reformulation of the chance constraints. In more detail, the formulation of the CCOPF problem considering the primary frequency response of generators is provided in section 2 and we propose, in section 3 a two-step distributed approach to solve this problem in a fully distributed manner. The first step aims to determine the sensitivity factors of the system, namely the Generalized Generation Distribution Factors that measure the line flow changes due to deviations from generation schedule. The results of this first step are inputs of the second step that is the CCOPF. Both steps are distributedly solved by the ADMM algorithm. Hence, this two-step algorithm ensures the privacy and autonomy of the different system actors and it is de facto parallel and adapted to high performance platforms. More details on the background on ADMM based approach to solve the OPF from [10] can be found in Appendix A and finally, the results on a 2-bus system and on the IEEE 14-bus test system are available in Appendix B.

\section{CHANCE-CONSTRAINED OPF WITH PFC FORMULATION}

This section presents the different types of devices ${ }^{1}$ we consider in this paper, namely, wind farms, chance-constrained dispatchable generators participating in the primary frequency control and chance-constrained lines. The CCOPF formulation presented is similar to that of [17], but with a focus on the OPF instead of on the SCOPF problem and an instantiation of the chance constraints to take into account the primary frequency control schemes.

\subsection{Wind farms}

Let $W \subset D$ be the set of wind farms, included in the set of devices $D$ of the system. For a wind farm $w \in W$, let $\mu_{w}$ be its forecast expected production. Since wind farms are variable and non-dispatchable sources there is a forecast error that models the uncertainty with respect to the expected production (i.e. imbalances are expected between the expected production and the actual production of the wind farm). Using the same reasoning as in [17], the forecast errors are represented as Gaussian random variables with zero mean:

$$
\forall w \in W, \Delta p_{w} \sim \mathcal{N}\left(0, \sigma_{w}^{2}\right)
$$

where the forecast of different wind farms are assumed to be spatially uncorrelated. ${ }^{2}$

\subsection{Chance-constrained generators}

In our formulation, dispatchable generators compensate the deviation of wind farms from the forecasts through the primary frequency control, described next in Section 2.2.1. Once introduced, we proceed to derive the probabilistic formulation of conventional generators in Section 2.2.2.

2.2.1 Primary frequency control modeling. Let $G \subset D$ be the set of generators of the system. The primary frequency control (PFC) aims at regulating the frequency of the power system by adapting the generation [4]. The contribution of a generator $g$ to primary response is determined by the ratio of its coefficient, $K_{g}$, and the equivalent speed droop of the system, $\sum_{g \in G} K_{g}$. In turn, the coefficient $K_{g}$ is defined as the ratio of the generator maximum active power $\left(\bar{p}_{g}\right)$ and the speed droop of the generator (both constant characteristics of the generators). Hence, the response of a generator to a deviation from the power scheduled, $\Delta p$, can be formally expressed by the following steady-state equation:

$$
\Delta p_{g}=\frac{K_{g}}{\sum_{g^{\prime} \in G} K_{g^{\prime}}} \Delta p
$$

2.2.2 Probabilistic formulation. The compensation of the forecast error of the wind farm is achieved by the generators following the primary frequency control described above. Thus, following Equation 2, the primary frequency response of a generator $g \in G$ results from the product of a constant and the aggregated forecast error of all wind farms in the system. The aggregated forecast

\footnotetext{
${ }^{1}$ Loads are omitted since their formulation does not change w.r.t. OPF

${ }^{2}$ This assumption holds when wind farms are sufficiently far away from each other.
} 
error of wind farms, i.e. $\Delta p=\sum_{w \in W} \Delta p_{w}$, turns into a sum of uncorrelated Gaussian variables, and hence $\Delta p \sim \mathcal{N}\left(0, \sum_{w \in W} \sigma_{w}^{2}\right)$.

Hence, the primary frequency response of a generator is also a random variable, following a normal distribution $\Delta p_{g} \sim \mathcal{N}\left(0, \sigma_{g}^{2}\right)$ with a variance correlated with the output power of wind farms as:

$$
\sigma_{g}^{2}=\left(\frac{K_{g}}{\sum_{g^{\prime} \in G} K_{g^{\prime}}}\right)^{2} \cdot \sum_{w \in W} \sigma_{w}^{2}
$$

Therefore, under this probabilistic model, the cost function of the generator is defined as the expectation of the deterministic quadratic cost function (see Eq. 23) applied on the total power generation output variable (i.e. the initial schedule production, $p_{g}$ plus the reserve $\mathrm{PFC}$ response):

$$
\mathbb{E}\left[f\left(p_{g}+\Delta p_{g}\right)\right]=\alpha \cdot \sigma_{g}^{2}+\alpha \cdot p_{g}^{2}+\beta \cdot p_{g}+\gamma
$$

Moreover, following the chance-constrained approach [17], we constrain that the maximum capacity limit of the generator is not reached given a certain tolerance to a violation $\epsilon$ :

$$
\mathbb{P}\left[p_{g}+\Delta p_{g} \leq \bar{p}_{g}\right]>1-\epsilon
$$

where $\mathbb{P}(\cdot)$ is the probability to respect the constraint on the generator production.

Under the assumption of Gaussianity, these constraints are simplified using $\Phi^{-1}(\cdot)$ is the quantile function of the standard gaussian distribution to become:

$$
p_{g}+\Phi^{-1}(1-\epsilon) \cdot \sigma_{g} \leq \bar{p}_{g}
$$

\subsection{Chance-constrained lines}

To formulate the chance-constraints on lines, we need to be able to evaluate the impact of the deviation of a wind farm from its forecast on the lines flows. We then first introduce the sensitivity factors that we use to formulate the lines sub-problems.

2.3.1 Generalized Generation Distribution Factors. The Generalized Generation Distribution factors (GGDFs), first introduced in [16], are used to determine the lines flow changes due to a deviation of power injection in the system. Let $L \subset D$ be the set of lines of the system. As we are considering that any deviation of power injection is linearly compensated by generators that participate in the primary frequency control, we can determine the GGDF of a line $l \in L$ due to the deviation from forecast $\Delta p_{w}$ of a wind farm $w \in W$, i.e. $G F_{l}^{w}$, through the equation:

$$
\Delta p_{l}^{w}=G F_{l}^{w} \cdot \Delta p_{w}
$$

Moreover the linearity of Eq. 7 allows the use of superposition, so that, for a set of wind farms $W$ that deviate from their forecast by $\forall w \in W, \Delta p_{w}$, we can determine the power flow change in line $l$ due to all those deviations with :

$$
\Delta p_{l}=\sum_{w \in W}\left(G F_{l}^{w} \cdot \Delta p_{w}\right)
$$

2.3.2 Probabilistic formulation. We use the GGDF to formulate the probabilistic optimal power flow, as in [17], by adding chance constraints in the line flow capacity constraints, as described next.

As for generators, the amount of power transmitted through a line is affected by primary response of generators to the uncertainty of the wind farms and the line flow changes is a random variable following a normal distribution:

$$
\Delta p_{l} \sim \mathcal{N}\left(0, \sigma_{l}^{2}\right)
$$

With the notation of the previous section on GGDF, we can determine the relationship between the variance on the lines flows and the variance of the wind farms :

$$
\sigma_{l}^{2}=\sum_{w \in W}\left(G F_{l}^{w} \cdot \sigma_{w}\right)^{2}
$$

The chance constraints applied on lines enforce that the line flow capacity $\bar{p}_{l}$ is not reached given a certain tolerance $\epsilon$ to violation:

$$
\mathbb{P}\left[-\bar{p}_{l} \leq p_{l_{1}}+\Delta p_{l} \leq \bar{p}_{l}\right]>1-\epsilon
$$

where $\mathbb{P}(\cdot)$ is the probability distribution of the amount of power transmitted through the line.

As for generators, under the assumption of Gaussianity, these constraints are simplified using $\Phi^{-1}(\cdot)$, the quantile function of the standard Gaussian distribution to become:

$$
-\bar{p}_{l} \leq p_{l_{1}}+\Phi^{-1}(1-\epsilon) \cdot \sqrt{\sum_{w \in W}\left(G F_{l}^{w} \cdot \sigma_{w}\right)^{2}} \leq \bar{p}_{l}
$$

\section{DISTRIBUTED ALGORITHMS}

This section presents the two-step distributed algorithm for solving the CCOPF problem with PFC formulated in previous section. First, we describe how we determine the sensitivity factors and the equivalent speed droop of the system. Then, we provide the ADMM updates for the CCOPF sub-problems.

\subsection{Step 1: Distributed computation of sensitivity factors}

Notice that to define lines and generators problems, and in particular Eq. 12 and 3, line agents need to compute the GGDF with respect the deviation of the wind farms, as defined in section 2.3.1, and conventional generators need to compute the equivalent speed droop $\sum_{g \in G} K_{g}$. We propose in this section a distributed protocol that determines the GGDF and the equivalent speed droop.

First the equivalent speed droop is determined by computing a distributed sum, using the efficient Push-Sum protocol [9].

To determine the GGDF of the lines, we need to solve an initial power flow with a feasible power injection schedule in order to get the line flows $\forall l \in L, p_{l}^{(0)}$ and power injection schedules $\forall g \in$ $G, p_{g}^{(0)}$, a base case scenario. We also need to create one case per wind farm, in which, we apply a deviation $\Delta p_{w}$ at one of the wind farm $w \in W$. We determine the new power injection schedule of conventional generators by spreading $\Delta p_{w}$, and with the equivalent speed droop of the system. We finally solve the power flow with this new injection schedule, for each line $l \in L$, we get $p_{l}^{w}$, the power flow of line $l$ associated with the wind farm power deviation $\Delta p_{w}$. Then, in each scenario that considers the deviation of one of the wind farms $w \in W$, we determine the GGDF, $G F_{l}^{w}$, of each line $l \in L$ by computing :

$$
\forall w \in W, G F_{l}^{w}=\frac{\Delta p_{l}^{w}}{\Delta p_{w}}=\frac{p_{l}^{(0)}-p_{l}^{w}}{\Delta p_{w}}
$$


In summary, this first step of the algorithm is divided into four different substeps that can be run by agents sequentially or in parallel:

1 Run a distributed sum algorithm to calculate the equivalent speed droop, i.e. $\sum_{g \in G} K_{g}$.

2 Determine a feasible power injection schedule $\left(\forall g \in G, p_{g}^{(0)}\right)$ and power flows $\left(\forall l \in L, p_{l}^{(0)}\right)$ with the ADMM.

3 Create for each wind farm $w \in W$ a scenario in which a deviation of $w\left(\Delta p_{w}\right)$ is applied and spread and in which the frequency response of each generator is computed based on that deviation $\left(\forall g \in G, p_{g}^{(w)}=p_{g}^{(0)}+\frac{K_{g}}{\sum_{g \in G} K_{g}} \cdot \Delta p_{w}\right)$.

4 Run the ADMM algorithm to compute the power flows in each of these scenarios $\left(\forall l \in L, \forall w \in W, p_{l}^{(w)}\right)$.

After the substep 4, each line agent has all information needed to compute its GGDF factor for each wind farm, i.e. $\forall w \in W, G F_{l}^{(w)}=$ $\frac{p_{l}^{(0)}-p_{l}^{(w)}}{\Delta p_{w}}$. Note that those steps can be computed in parallel and that we can use previous power flow solutions as base case (step 2) and as warm starts to carry out the distributed power flow (step 4).

\subsection{Step 2: Distributed CCOPF}

The outputs from the sensitivity analysis are now the input of CCOPF problem. We provide a description of the sub problems implemented in devices and nets agents after applying the ADMM.

3.2.1 Net agents. Before updating the scaled dual variables following Eq. 21-22, each net agent solves its sub-problem, Eq. 20. Since nets agents are not different from [10], the ADMM updates are the same than the ones provided in [10], namely, for any net $n \in N$, at iteration $k+1$ :

$$
\dot{p}_{n}^{k+1}=p_{n}^{k+1}-\frac{1}{|n|} \cdot \sum_{d \in n} p_{d}^{k+1}, \quad \dot{\theta}_{n}^{k+1}=\frac{1}{|n|} \cdot \sum_{d \in n} \theta_{d}^{k+1}
$$

3.2.2 Wind farm agents. The mean power injections of the wind farms are constant, there is nothing to optimize. However, the wind farms spread the value of their variance $\sigma_{w}^{2}$ in a peer to peer fashion

3.2.3 Chance-constrained generator agents. Each chance constrained generator $g \in G$ solves, at iteration $k+1$, the sub-problem:

$$
\begin{gathered}
\min _{p_{g}} \alpha \cdot \sigma_{g}^{2}+\alpha \cdot p_{g}^{2}+\beta \cdot p_{g}+\gamma+\frac{\rho}{2}\left\|\dot{p}_{g}^{k}-u_{g}^{k}-p_{g}\right\|_{2}^{2} \\
\text { S.t. } \quad \sigma_{g}=\frac{K_{g}}{\sum_{g^{\prime} \in G} K_{g^{\prime}}} \cdot \sqrt{\sum_{w \in W} \sigma_{w}^{2}} \\
r_{g}=\Phi^{-1}(1-\epsilon) \cdot \sigma_{g} \quad p_{g}+r_{g} \leq \bar{p}_{g}
\end{gathered}
$$

The solution to this problem without the constraints is simple as it is just a polynomial of order 2 . When the unconstrained solution does not meet the constraint, it means that the inequality becomes an equality and the problem is even simpler, [19].

3.2.4 Chance-constrained line agents. We present the chance constrained sub-problem solved by lines, keeping in mind that we determined the GGDF, related to each wind farm, of the lines through the distributed sensitivity analysis. We then have :

$$
\begin{aligned}
& \min _{\left(p_{l_{1}}, \theta_{l_{1}}, p_{l_{2}}, \theta_{l_{2}}\right)} \quad \frac{\rho}{2}\left\|\dot{p}_{l_{1}}^{k}-u_{l_{1}}^{k}-p_{l_{1}}\right\|_{2}^{2}+\frac{\rho}{2}\left\|\dot{p}_{l_{2}}^{k}-u_{l_{2}}^{k}-p_{l_{2}}\right\|_{2}^{2} \quad \text { (16a) } \\
&+\frac{\rho}{2}\left\|\dot{\theta}_{l_{1}}^{k}-v_{l_{1}}^{k}-\theta_{l_{1}}\right\|_{2}^{2}+\frac{\rho}{2}\left\|\dot{\theta}_{l_{2}}^{k}-v_{l_{2}}^{k}-\theta_{l_{2}}\right\|_{2}^{2} \quad \text { (16b) } \\
& \text { S.t. } \quad p_{l_{1}}=-p_{l_{2}}=b \cdot\left(\theta_{l_{2}}-\theta_{l_{1}}\right), \quad-\bar{p}_{l} \leq p_{l_{1}}+r_{l} \leq \bar{p}_{l} \quad \text { (16c) } \\
& \sigma_{l}=\sqrt{\sum_{w \in W}\left(G F_{l}^{w} \cdot \sigma_{w}\right)^{2}}, \quad r_{l}=\Phi^{-1}(1-\epsilon) \cdot \sigma_{l} \quad(16 \mathrm{~d})
\end{aligned}
$$

We solve the line flow problem only considering the equality constraints without the inequality constraints, as in [19], and then check that the inequality constraint is satisfied. If one of the constraints is not respected, let's say the solution $p_{l_{1}}^{*}$ is so that $p_{l_{1}}^{*}+\Phi^{-1}(1-\epsilon) \cdot \sigma_{l} \geq \bar{p}_{l}$, we then apply the equality constraint $p_{l_{1}}=-\Phi^{-1}(1-\epsilon) \cdot \sigma_{l}+\bar{p}_{l}$ and only solve the problem on the voltage phase angles.

3.2.5 Loads. The loads are considered constant, the sub-problem solution is fixed.

\section{SIMULATIONS}

The proposed distributed algorithm was implemented and tested on a two-bus test system and on the IEEE 14-bus test system; the results of these simulations are provided in Appendix B. The ability of the proposed algorithm to accurately determine the GGDF and solve CCOPF problem with no central coordination is validated. Moreover, the results show that solving the second step of the algorithm does not necessitate significantly more iterations than solving the OPF problem.

\section{CONCLUSION AND FUTURE WORK}

In this paper, we propose a two-step algorithm that solves, with distributed methods, the Chance-Constrained Optimal Power Flow problem (CCOPF) based on an exact reformulation of the problem assuming that wind power forecast errors follow independent Gaussian distributions.

The proposed method employs a distributed solution to cope with the inter-regional OPF problem, while previous works (i.e. $[2,11,17])$ need to adopt centralized strategies for both the computation of the sensitivity factors and the CCOPF solution. The distributivity ensures the privacy and autonomy of the actors of interconnected systems and enables the parallelization of the computation. The first step of the algorithm aims at determining the generalized generation distribution factors and the equivalent speed droop of the system, when the second step is the distributed CCOPF solution in its self. We provide simulation results on a two-bus test system and on the IEEE 14-bus test system, and show the ability of this algorithm to solve our problem.

As a future work, we plan to extend this distributed framework to consider other non-Gaussian distributions to model the wind forecast errors, e.g. Weibull distributions. Another natural extension would be to consider the probabilistic security-constrained optimal power flow that considers potential contingencies on devices of the system, in addition to the uncertainty on some elements. 


\section{ACKNOWLEDGMENTS}

M. Vinyals was supported by the European Union under the FP7, and by Horizon 2020 research and innovation program, Grant Agreements No. : 619682 and No. : 77443.

\section{A BACKGROUND}

We review in this section the methodology for decomposing the OPF problem and its mapping to the ADMM algorithm from [10] as well as the objective function of the main components of interest in the OPF problem. The models use the DC power flow equations (linearized) and thus the variables of the system are active power injections or flows, denoted as $p$, or voltage phase angles at the buses, denoted as $\theta$.

\section{A.1 OPF network decomposition and ADMM}

Given a power system, the network decomposition from [10] classifies the power system components into two sets: (i) the set of nets $(N)$, that similar to the electrical bus concept connect devices and enforce Kirchhoff's physical laws and phase consistency; and (ii) the set of devices $(D)$, that is composed of all power components that are not buses. Each component $c \in D \cup N$ is associated with a local objective function $\left(f_{c}(\cdot)\right)$ and local constraints $\left(C_{c}\right)$ that apply on a set of local variables $\left(x_{c}\right)$. Considering the DC power flow equations (linearized), the local set of variables will contain the corresponding two types of variables: $x_{c}=\left(p_{c}, \theta_{c}\right)$. For the sake of concision, we say that $f_{c}\left(x_{c}\right)=\infty$ if $x_{c} \notin C_{c}$, otherwise $f_{c}\left(x_{c}\right)$ represents the cost associated with the set of variables $x_{c}$ for the component $c$. Now, we create an edge for every pair of components whose objective function have some variable in common (i.e. the cost and/or the feasibility of both components depends on at least some shared variables). We will refer to this set of edges as terminals $(T)$. For each component $c \in N \cup D$, we use $c$ to refer to both the component itself as well as to the set of neighbours associated with it, i.e., we say $c^{\prime} \in c$ if component $c^{\prime}$ is linked with component $c$ by means of a terminal. Accordingly, $|c|$ is the number of components connected to $c$.

Under this model, the global objective function of the OPF problem can be expressed as the sum of local objective functions:

$$
\min _{p, \theta} \sum_{d \in D} f_{d}\left(p_{d}, \theta_{d}\right)+\sum_{n \in N} f_{n}\left(p_{n}, \theta_{n}\right)
$$

The global objective function is intended to find the active power and voltage phase angles that minimize the overall operating cost while satisfying the power flow equations. Basically, nets objective functions encodes the Kirchhoff's laws and phase consistency, while devices objective functions include the cost of generation and local constraints on the devices.

Following [10], this optimization problem can be solved by a message-passing protocol based on the Alternating Direction Method of Multipliers (ADMM) [3]. Under ADMM formulation, first, the nets objective functions are defined over a duplicated copy of the original variables (i.e. denoted as $\dot{p}, \dot{\theta}$ ) and equality constraints $(p=\dot{p}, \theta=\dot{\theta})$ are added to keep the equivalence with Eq. 17 .
The scaled form of the augmented Lagrangian is then formed by relaxing the equality constraints:

$$
\begin{aligned}
L(p, \dot{p}, \theta, \dot{\theta}, u, v)= & \sum_{d \in D} f_{d}\left(p_{d}, \theta_{d}\right)+\sum_{n \in N} f_{n}\left(\dot{p}_{n}, \dot{\theta}_{n}\right) \\
& +\frac{\rho}{2}\left(\|p-\dot{p}+u\|_{2}^{2}+\|\theta-\dot{\theta}+v\|_{2}^{2}\right)
\end{aligned}
$$

where $\rho>0$ is the scaling parameter, $u$ and $v$ are the dual variables associated, respectively, with the active power schedule $p$ and the voltage angle schedule $\theta$.

Following [10], the local problems then become separable using the fact that set of devices $D$ and the set of nets $N$ are both partitions of the set of terminals $T$, and we can apply the ADMM algorithm that consists in the following three-step iterative process:

The device-minimization step (i.e. parallelized among devices):

$$
\begin{gathered}
\left(p_{d}^{k+1}, \theta_{d}^{k+1}\right)=\arg \min _{p_{d}, \theta_{d}}\left(f_{d}\left(p_{d}, \theta_{d}\right)+\right. \\
\left.\frac{\rho}{2}\left\|p_{d}-\dot{p}_{d}^{k}+u_{d}^{k}\right\|_{2}^{2}+\frac{\rho}{2}\left\|\theta_{d}-\dot{\theta}_{d}^{k}+v_{d}^{k}\right\|_{2}^{2}\right), \quad \forall d \in D
\end{gathered}
$$

The net-minimization step (i.e. parallelized among nets):

$$
\begin{gathered}
\left(\dot{p}_{n}^{k+1}, \dot{\theta}_{n}^{k+1}\right)=\underset{\dot{p}_{n}, \dot{\theta}_{n}}{\arg \min }\left(f_{n}\left(\dot{p}_{n}, \dot{\theta}_{n}\right)+\right. \\
\left.\frac{\rho}{2}\left\|p_{n}^{k+1}-\dot{p}_{n}+u_{n}^{k}\right\|_{2}^{2}+\frac{\rho}{2}\left\|\theta_{n}^{k+1}-\dot{\theta}_{n}+v_{n}^{k}\right\|_{2}^{2}\right), \quad \forall n \in N
\end{gathered}
$$

The (price) scaled dual variables update (i.e. parallelized among nets):

$$
\begin{aligned}
& u_{n}^{k+1}=u_{n}^{k}+\left(p_{n}^{k+1}-\dot{p}_{n}^{k+1}\right), \quad \forall n \in N \\
& v_{n}^{k+1}=v_{n}^{k}+\left(\theta_{n}^{k+1}-\dot{\theta}_{n}^{k+1}\right), \quad \forall n \in N
\end{aligned}
$$

The problem is, by construction, already separated in local subproblems which allows each agent (either net or device) to solve its sub-problem in parallel and to coordinate via message-passing through terminals. Hence, at each iteration, each device agent computes a minimization step for its local objective function (Eq. 19) that minimizes its operating cost (i.e. encoded by $f_{d}$ ), and a penalty that depends on messages passed to it through its terminals by its neighboring nets in the previous iteration $\left(\dot{p}_{n}^{k+1}, \dot{\theta}_{n}^{k+1}, u_{n}^{k+1}\right.$ and $v_{n}^{k+1}$ ). Similarly, each net component computes its minimization (Eq. 20) and scaled dual variables update steps (Eq. 21 and 22) with an argument that depends on messages passed to it through its terminals by its neighboring devices in the previous iteration $\left(p_{n}^{k+1}, \theta_{n}^{k+1}\right)$. This is done iteratively until a sufficient consistency is reached at each net.

$\mathrm{ADMM}$ is guaranteed to converge to the optimal solution when all devices have convex, closed, proper objective functions and a feasible solution to the problem exists.

\section{A.2 Objective functions of nets and main devices}

We present, in this section, the devices and the nets objective functions mentioned in Eq. 17, that were previously presented and solved in $[10,19]$. We consider generators, loads, lines and nets. 
A.2.1 Dispatchable generators. A dispatchable generator $g$ injects on request some power $p_{g}$ to the system at a cost determined by its quadratic objective function :

$$
f\left(p_{g}\right)=\alpha \cdot p_{g}^{2}+\beta \cdot p_{g}+\gamma
$$

where $\alpha, \beta$ and $\gamma$ are respectively quadratic, linear and constant cost coefficients.

Moreover, the power output of the generator has to remain within its production limits, i.e. $p_{g} \leq \bar{p}_{g}$ where $\bar{p}_{g}$ is the maximum power output.

A.2.2 Lines. A line incurs no cost by itself, but it must enforce the power flow equations, so that, for line $l$, with susceptance $b_{l}$, we have :

$$
p_{l_{1}}=-p_{l_{2}}=b_{l} \cdot\left(\theta_{l_{2}}-\theta_{l_{1}}\right)
$$

with $\theta_{l_{1}}$ and $\theta_{l_{2}}$ the voltage phase angles respectively at buses $l_{1}$ and $l_{2}$, and, $p_{l_{1}}$ and $p_{l_{2}}$ the power flows from $l_{1}$ and $l_{2}$ respectively.

Moreover, the power flows have to remain within the transmission limits, i.e. $-\bar{p}_{l} \leq p_{l_{1}} \leq \bar{p}_{l}$ where $\bar{p}_{l}$ is the maximum capacity of the line.

A.2.3 Loads. Loads are assumed to consume a fixed amount, providing no flexibility or further constraints.

A.2.4 Nets. Each net $n \in N$ requires power balance and phase consistency to be enforced as follows:

$$
\begin{array}{r}
\sum_{d \in n} \dot{p}_{d}=0 \\
\forall d, d^{\prime} \in n, \quad \theta_{d}-\theta_{d^{\prime}}=0
\end{array}
$$

\section{B SIMULATION RESULTS}

The proposed distributed algorithm was implemented in the Java Agent DEvelopment ${ }^{3}$ (JADE) platform [1], where each agent solves its corresponding sub-problem in parallel. We tested our algorithm on two power systems: a small 2-bus test system to illustrate its operation and the IEEE 14-bus test system for validation on a realistic system. For each circuit, we tested it under scenarios with different percentages of wind forecast error, where the percentage forecast error is defined by setting the standard deviation (i.e. the square root of the variance) of the wind error distribution to a percentage of its mean value (e.g. a 10\% of forecast error fir a wind farm with an expected value of $100 \mathrm{MW}$ leads to an error prediction distribution with a variance of $10^{2}$ ). For each scenario we enforce that the reserves and margins are sufficient in $99.7 \%$ of the time, i.e. $\phi^{-1}(0.997)=3$.

\section{B.1 2-bus test system}

We tested our algorithm on a 2-bus test system with two conventional generators, a line, a load and a wind farm, as depicted in Figure 1a. The two conventional generators are identical (same capacity limits, same coefficients i.e. $K_{G_{1}}=K_{G_{2}}=K_{G}$ ), except that the cost of generation of $G_{1}$ is cheaper than $G_{2}$. We selected this circuit because it is simple enough to allow for an analytical solution.

First, we focus on the solution of the OPF problem (i.e. the one that minimizes the cost of operation and enforces the line and

\footnotetext{
3 jade.tilab.com
}

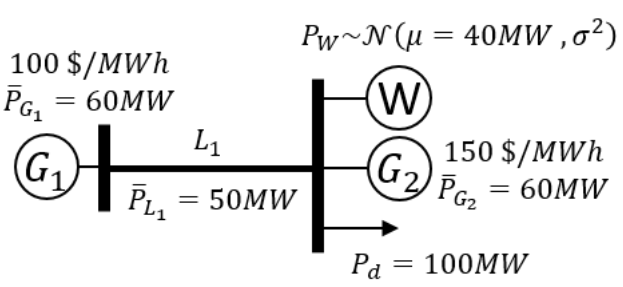

(a) 2-bus test system.

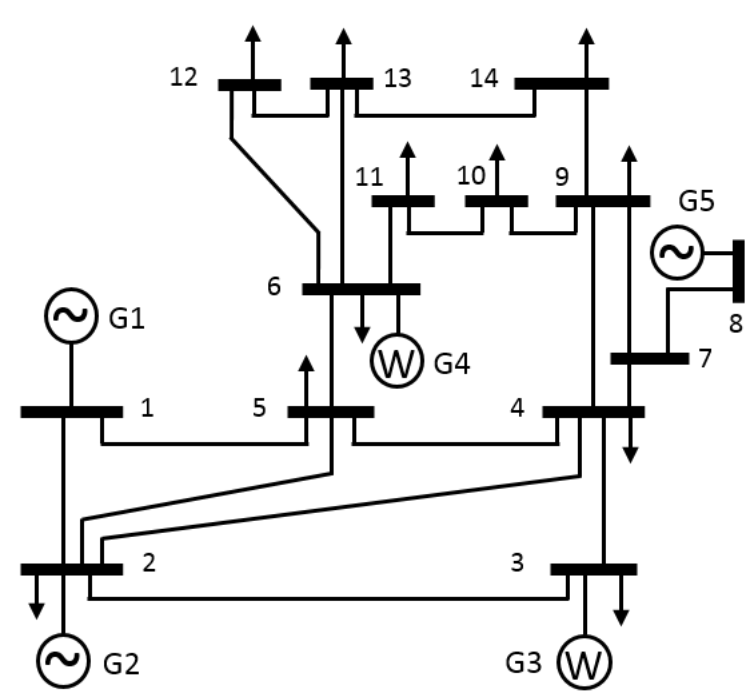

(b) IEEE 14-bus test system.

Figure 1: Test systems.

generators constraints). The wind farm in this case is supposed to generate its mean power $\mu$, i.e. $40 \mathrm{MW}$. To minimize the cost, we need to maximize the use of generator $G_{1}$ (cheaper than $G_{2}$ ), but we also must ensure that the line $L_{1}$ is not overloaded. $G_{1}$ then generates the maximum power the line can carry $\bar{p}_{L_{1}}$, i.e. $50 \mathrm{MW}$, and finally $G_{2}$ covers the remaining power needed, i.e. $10 \mathrm{MW}$.

Second, we consider the uncertainty in the wind farm forecast and the case in which the generators $G_{1}$ and $G_{2}$, and line $L_{1}$ enforce that they have sufficient reserves or margins to cover most of the wind farm deviations.

See that, in this simple example, there is only one line that transmits all the power generated by generator $G_{1}$ to the rest of the system, i.e. bus 2. The GGDF reflects the impact of a deviation of the wind farm on the line flow and thus, on the generation of generator $G_{1}$. Then from Eq. 2, we get :

$\Delta p_{L_{1}}=\Delta p_{G_{1}}=\frac{K_{G}}{K_{G}+K_{G}} \cdot \Delta p_{w}=\frac{1}{2} \cdot \Delta p_{w}=G F_{L_{1}}^{w} \cdot \Delta p_{w}$.

The standard deviation for the (equal) generators is calculated using Eq. $15 \mathrm{~b}$ as $\sigma_{G_{1}}=\sigma_{G_{2}}=\frac{K_{G}}{K_{G}+K_{G}} \cdot \sigma_{w}=\frac{1}{2} \cdot \sigma_{w}$ whereas for lines is computed through Eq. $16 \mathrm{~d}$ as $\sigma_{L_{1}}=G F_{l}^{w} \cdot \sigma_{w}$. Then the reserves and margins are defined in Eq. $15 \mathrm{c}$ and Eq. $16 \mathrm{~d}$ so that $r_{G_{1}}=r_{G_{2}}=\frac{3}{2} \cdot \sigma_{w}$ and $r_{L_{1}}=\frac{3}{2} \cdot \sigma_{w}$. 


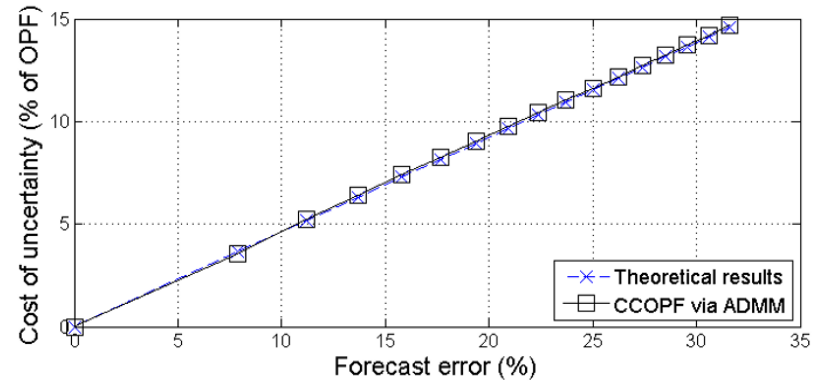

(a) Percentage error compare to theory.

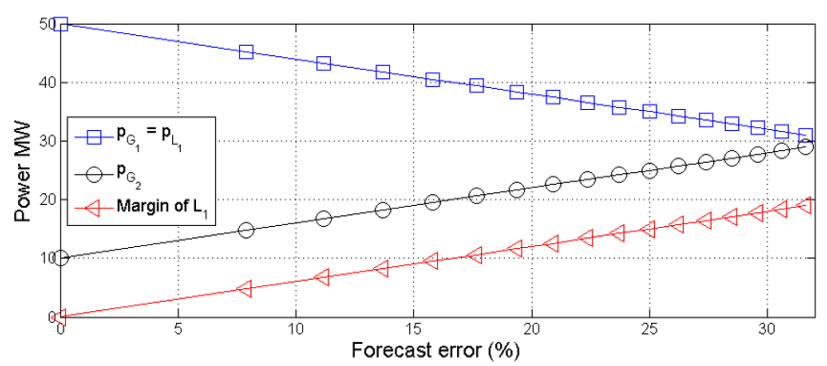

(b) Power scheduled in generators, in the line and the margin needed for the line.

Figure 2: Results on the 2-bus test system.

The limiting constraint, in this example, is the capacity of line $L_{1}$ because all the power generated by $G_{1}$ is flowing through $L_{1}$ that have lower capacity than $G_{1}$. It follows that the scheduled power output of generator $G_{1}$ is $p_{G_{1}}=\bar{p}_{L_{1}}-\frac{3}{2} \cdot \sigma_{w}$, and as this power can only be transmitted through line $L_{1}$, the expected power flow in line $L_{1}$ is equal to $p_{G_{1}}$. Generator $G_{2}$ covers the rest of power needed to fulfill the load consumption $p_{G_{2}}=\bar{p}_{L_{1}}-\frac{3}{2} \cdot \sigma_{w}+\mu_{w}-p_{d}$.

We propose to compare the theoretical results, provided above, with the results obtained with our algorithm using a scaling parameter $\rho=1$ and a tolerance equal to $10^{-4}$.

Figure 2a depicts the cost of uncertainty, that represent the cost increase when considering the uncertainty on the wind production, for the analytical solution and for the two-step ADMM algorithm when varying the wind forecast error. The cost of uncertainty found by our algorithm matches well the analytical results, and the relative error of our results compared to the analytical solution is below $0.1 \%$ for all cases. It took 50 iterations for the ADMM algorithm to solve the OPF and between 57 and 59 iterations (i.e. depending on the particular wind forecast error) to find the solutions of the CCOPF, which shows that the addition of the chance constraints do not increase significantly the number of iterations needed.

Figure $2 \mathrm{~b}$ depicts the generation of both generators and the margin of line $L_{1}$. We observe that when the uncertainty grows, the margin needed increases and thus the power generation is more and more scheduled on generator $G_{2}$ which increases the cost of operations.

\begin{tabular}{|c|c|c|c|c|}
\hline Gen. & $\begin{array}{c}\bar{p} \\
(\mathrm{MW})\end{array}$ & $\begin{array}{c}\mathrm{p} \\
(\mathrm{MW})\end{array}$ & $\begin{array}{c}\beta \\
\left(\$ / M W h^{2}\right)\end{array}$ & $\begin{array}{c}\gamma \\
(\$ / \mathrm{MWh})\end{array}$ \\
\hline$G 1$ & 332.4 & 0 & 0.043 & 20 \\
\hline$G 2$ & 140 & 0 & 0.25 & 20 \\
\hline$G 5$ & 100 & 0 & 0.01 & 40 \\
\hline
\end{tabular}

Table 1: Generators parameters used in the IEEE 14-bus test system.

\section{B.2 IEEE 14-bus test system}

This section tests the performance of the proposed algorithm on the IEEE-14-bus test system with the transmission data from the Power System Test Case Archive ${ }^{4}$, where we replace the generators on bus $3\left(G_{3}\right)$ and on bus $6\left(G_{4}\right)$ by identical wind farms. The system, represented in Figure 1b, is composed of 11 loads, 20 lines, 3 conventional generators and 2 wind farms. We completed the model by setting the line capacity limits to $50 \mathrm{MW}$, except for lines 1-2, 1-5, 2-3, 7-8 and 7-9 that were set to $110 \mathrm{MW}$ because those lines carry most of the power of the system. Table 1 details the parameters used for the different generators. We compare the results obtained with our algorithm with the solution found by a centralized solver using MATLAB [15] and CVX a package for solving convex programs $[5,6]$. Depending on the step of the algorithm, we use different scaling parameters but we set for all ADMM processes a tolerance equal to $10^{-4}$.

As the first substep s1.1 of the algorithm, agents compute the equivalent speed droop of the system distributedly, in 25 iterations, via the Push-Sum algorithm. In the substep s1.2, agents run ADMM to calculate an optimal power flow in a distributed manner. This initial feasible point was found in 761 iterations with a scaling parameter $\rho=1$. This solution is then reused by agents as a warm start to solve the scenarios in which the wind farms deviate (substep s1.3), i.e. the power flow variables are initialized to the previously calculated solution. With two wind farms, this means to solve two power flows with deviations of the power injection schedule ruled by a shift of a wind farm and the primary frequency control. Solving these two scenarios in parallel by ADMM with a scaling parameter set to $\rho=10^{-2}$ took 556 iterations until convergence.

Hence, it took a total of 1342 iterations for the agents to compute the GGDF in a distributed manner. The results of the first step of the proposed algorithm are summarized and compared in Figure 3. We compare the GGDF obtained, for each wind farm (G3 and G4) and at each line, from our distributed algorithm with those from the reference calculated in a centralized manner. The lines most impacted by generator $G_{3}$ are lines 1-2, 2-3 and 3-4, when the most impacted by $G_{4}$ are lines 1-2, 5-6 and 7-9, then these lines see their capacity shrinks more than the other lines of the system when the uncertainty grows.

Note that this first step needs to be computed again only if the generators connected or the topology change and that we could have used a previous power flow solution instead of running an OPF in substep s1.2.

\footnotetext{
${ }^{4}$ https://www2.ee.washington.edu/research/pstca/
} 


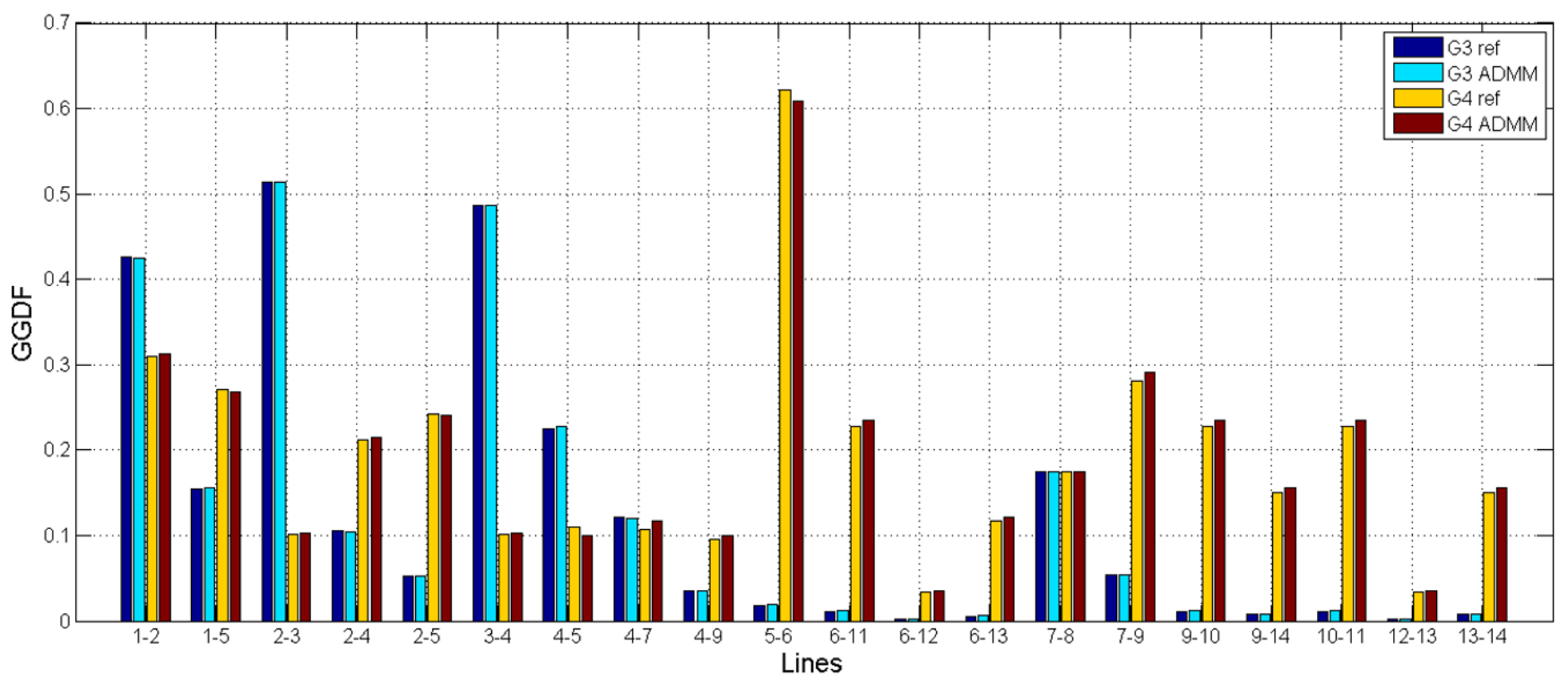

Figure 3: IEEE 14-bus GGDF and relative error compared to centralized calculation.

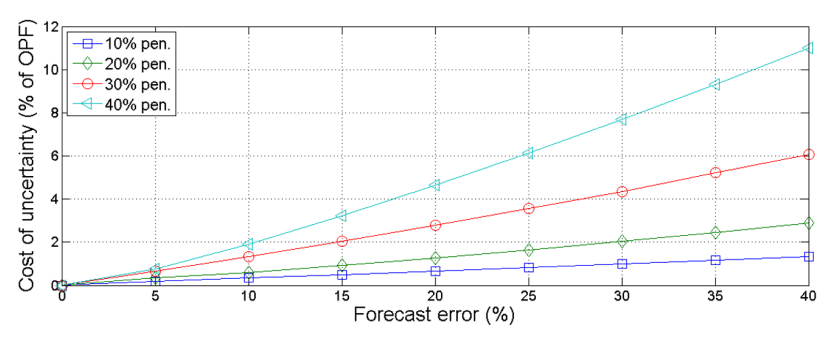

(a) Comparison of theoretical results and via our method based on ADMM.

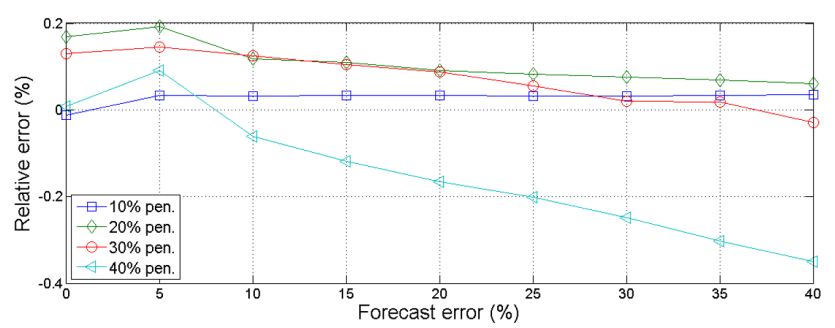

(b) Percentage error compare to theory.

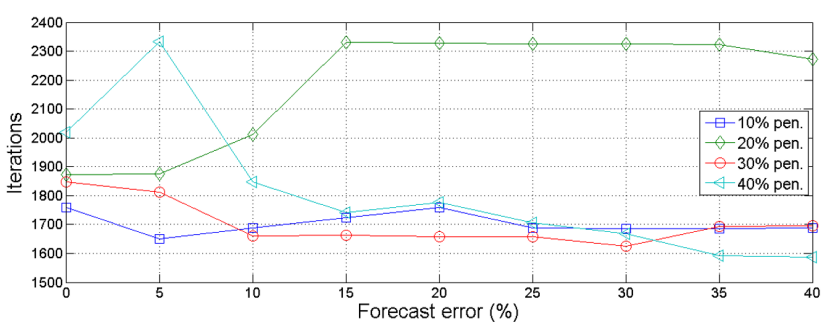

(c) Iterations needed to reach convergence.

Figure 4: Results on the 14-bus test system.
Finally, for the second step of the algorithm we perform simulations with different wind penetrations levels (the term wind penetration level is defined here as the ratio of the wind production and the total consumption of the system) and with different wind forecast errors. In particular, we used $10 \%, 20 \%, 30 \%$ and $40 \%$ wind penetration levels and we vary the percentage forecast error between $0 \%$ (no forecast error, i.e. OPF) and $40 \%$ The scaling parameter for ADMM in this case was set to $\rho=10$.

We present the cost of uncertainty, in Figure 4a, defined as the cost increase in percentage of the OPF solution, for each wind penetration level and for different forecast errors. Observe that as the forecast error grows, the margin and reserves needed increase which leads to higher costs. Under a wind penetration level as low as $10 \%$, the impact of the uncertainty on the cost of operation is limited, even with a $40 \%$ forecast error does not exceed $1.4 \%$. However, with high wind penetration and a forecast error of $40 \%$, the cost of security goes up to $11 \%$ of the OPF cost of operation.

Figure $4 \mathrm{~b}$ presents the relative error of our simulation compared to the reference. The error is below $0.2 \%$ and is more conservative than the reference for wind penetration below $40 \%$. For the case with a wind penetration of $40 \%$, the underestimation can be explained by the fact that, depending on the wind penetration, different constraints imposes the power injection schedule. In the $40 \%$ wind penetration case, line $4-5$ is the limiting line and this line GGDF is underestimated compared to the reference for generator $G_{4}$.

Finally, the number of iterations for each case is presented in Figure $4 \mathrm{c}$. The number of iterations needed does not vary significantly and is not greater than the number of iterations to solve the optimal power flow (the OPF is $0 \%$ forecast error).

\section{REFERENCES}

[1] Fabio Bellifemine, Federico Bergenti, Giovanni Caire, and Agostino Poggi. 2005. JADE - A Java Agent Development Framework. In Multi-Agent Programming: 
Languages, Platforms and Applications. 125-147.

[2] Daniel Bienstock, Michael Chertkov, and Sean Harnett. 2014. Chance-constrained optimal power flow: Risk-aware network control under uncertainty. SIAM Rev. 56, 3 (2014), 461-495.

[3] Stephen Boyd, Neal Parikh, Eric Chu, Borja Peleato, and Jonathan Eckstein. 2011 Distributed Optimization and Statistical Learning via the Alternating Direction Method of Multipliers. Foundations and Trends in Machine Learning 3, 1 (2011), 1-122. https://doi.org/10.1561/2200000016

[4] Mircea Eremia and Mohammad Shahidehpour. 2013. Handbook of electrical power system dynamics: modeling, stability, and control. Vol. 92. John Wiley \& Sons.

[5] Michael Grant and Stephen Boyd. 2008. Graph implementations for nonsmooth convex programs. In Recent Advances in Learning and Control, V. Blondel, S. Boyd, and H. Kimura (Eds.). Springer-Verlag Limited, 95-110. http://stanford.edu/ boyd/graph_dcp.html.

[6] Michael Grant and Stephen Boyd. 2014. CVX: Matlab Software for Disciplined Convex Programming, version 2.1. http://cvxr.com/cvx. (March 2014).

[7] Ali Hassan, Yury Dvorkin, Deepjyoti Deka, and Michael Chertkov. 2017. Chanceconstrained ADMM approach for decentralized control of distributed energy resources. arXiv preprint arXiv:1710.09738 (2017).

[8] Amin Kargarian, Javad Mohammadi, Junyao Guo, Sambuddha Chakrabarti, Masoud Barati, Gabriela Hug, Soummya Kar, and Ross Baldick. 2016. Toward distributed/decentralized DC optimal power flow implementation in future electric power systems. IEEE Transactions on Smart Grid (2016)

[9] David Kempe, Alin Dobra, and Johannes Gehrke. 2003. Gossip-based computation of aggregate information. In Foundations of Computer Science, 2003. Proceedings. 44th Annual IEEE Symposium on. IEEE, 482-491.

[10] Matt Kraning, Eric Chu, Javad Lavaei, and Stephen P. Boyd. 2014. Dynamic Network Energy Management via Proximal Message Passing. Foundations and Trends in Optimization 1, 2 (2014), 73-126.

[11] Bowen Li and Johanna L Mathieu. 2015. Analytical reformulation of chanceconstrained optimal power flow with uncertain load control. In PowerTech, 2015 IEEE Eindhoven. IEEE, 1-6.

[12] Zhigang Li, Mohammad Shahidehpour, Wenchuan Wu, Bo Zeng, Boming Zhang, and Weiye Zheng. 2015. Decentralized multiarea robust generation unit and tieline scheduling under wind power uncertainty. IEEE Transactions on Sustainable Energy 6, 4 (2015), 1377-1388.

[13] Z. Li, W. Wu, B. Zeng, M. Shahidehpour, and B. Zhang. 2016. Decentralized Contingency-Constrained Tie-Line Scheduling for Multi-Area Power Grids. IEEE Transactions on Power Systems (2016), 1-14. https://doi.org/10.1109/TPWRS.2016. 2539278

[14] Kostas Margellos, Paul Goulart, and John Lygeros. 2014. On the Road Between Robust Optimization and the Scenario Approach for Chance Constrained Optimization Problems. IEEE Trans. Automat. Contr. 59, 8 (2014), 2258-2263. https://doi.org/10.1109/TAC.2014.2303232

[15] MATLAB. 2014. version 7.10.0 (R2014a). The MathWorks Inc., Natick, Massachusetts.

[16] Wai Y Ng. 1981. Generalized generation distribution factors for power system security evaluations. IEEE Transactions on Power Apparatus and Systems 3 (1981) 1001-1005.

[17] Line Roald, Frauke Oldewurtel, Thilo Krause, and Goran Andersson. 2013. Analytical reformulation of security constrained optimal power flow with probabilistic constraints. PowerTech (POWERTECH) (2013).

[18] Paul Scott and Sylvie Thiébaux. 2015. Distributed Multi-Period Optimal Power Flow for Demand Response in Microgrids. In Proceedings of the 2015 ACM Sixth International Conference on Future Energy Systems, e-Energy 2015, Bangalore, India, fuly 14-17, 2015. 17-26.

[19] Maxime Velay, Meritxell Vinyals, Yvon Besanger, and Nicholas Retière. 2017 Agent-based Security Constrained Optimal Power Flow with primary frequency control. In EUMAS 2017 - Proceedings of the Fiftheen European Workshop on MultiAgent Systems, Évry, France, December 14-15, 2017. To appear.

[20] Yamin Wang, Shouxiang Wang, and Lei Wu. 2017. Distributed optimization approaches for emerging power systems operation: A review. Electric Powe Systems Research 144 (2017), 127-135. https://doi.org/10.1016/j.epsr.2016.11.025 